\title{
Radiopharmaceuticals in paraganglioma imaging: too many members on board?
}

\author{
David Taïeb $^{1}$ - Rodney J. Hicks ${ }^{2,3} \cdot$ Karel Pacak $^{4}$
}

Received: 25 September 2015 / Accepted: 1 October 2015/Published online: 13 October 2015

(C) Springer-Verlag Berlin Heidelberg 2015

Since the development of radioiodinated metaiodobenzylguanidine (MIBG) over 30 years ago, nuclear imaging has had a central role in managing patients with paraganglioma (PGL), aiding in the diagnosis and staging of this rare but, for many patients, devastating disease [1]. This role is complemented by anatomical imaging, including computed tomography (CT) and magnetic resonance imaging (MRI). The major advantage of nuclear imaging is in providing high visual contrast between tumor and healthy tissue, which enables the detection of tumors that could potentially be missed by conventional imaging. Beyond its localization value, this imaging modality provides unique opportunities for better characterizing these tumors at molecular levels (e.g., catecholamine synthesis, specific receptor and transporter expression), mirroring ex vivo histological classification but on a whole-body, in vivo, scale. This opportunity has more recently been augmented by a number of excellent radiopharmaceuticals, which target different functional and molecular pathways that often reflect the diverse

David Taïeb

david.taieb@ap-hm.fr

1 Department of Nuclear Medicine, La Timone University Hospital, European Center for Research in Medical Imaging, Aix-Marseille University, 264 rue Saint-Pierre, 13385 Marseille, France

2 Cancer Imaging and Neuroendocrine Tumour Service, Peter MacCallum Cancer Centre, Melbourne, Australia

3 The Sir Peter MacCallum Department of Oncology, University of Melbourne, Melbourne, Australia

4 Program in Reproductive and Adult Endocrinology, Eunice Kennedy Shriver National Institute of Child Health \& Human Development (NICHD), National Institutes of Health, Bethesda, MD 20892, USA genetic landscape of PGL. Based on these characteristics, nuclear imaging provides a means of linking imaging phenotype to genotype and can be considered a member of the multi-omics approach. For example, an intense 2fluoro-2-deoxy-D-glucose $\left({ }^{18} \mathrm{~F}\right.$-fluorodeoxyglucose, ${ }^{18} \mathrm{~F}$ FDG) uptake by a PGL is mostly associated with mutations involving one of the genes encoding the succinate dehydrogenase (SDH) complex. Conversely, a low uptake can often rule out a classic SDH deficiency linked to SDH mutations [2]. In addition to genetic mutation, epigenetic mechanisms (histones and methylation modifications) and the tissue of origin may also impact the imaging phenotype. For example, whereas PGLs associated with the sympathetic nervous system often exhibit high ${ }^{18}$ F-FDG uptake, parasympathetic PGLs (typically arising in the head and neck region) may have very low uptake values. The ${ }^{18}$ F-FDG uptake pattern SDHxPGLs reflects metabolic reprogramming, a hypermethylator phenotype, and abnormal mitochondrial respiratory function [3] and it is predominantly linked with high succinate and low fumarate metabolomic pattern [4]. Such tumors are classified as exhibiting "pseudohypoxia." There are further differences based on the site of origin. For example, parasympatheticassociated PGLs almost always have an intense uptake of 6-fluoro-3,4-dihydroxyphenylalanine $\left({ }^{18} \mathrm{~F}\right.$-fluorodopa, ${ }^{18} \mathrm{~F}$-FDOPA) or somatostatin analogs (SSA) labeled with ${ }^{68} \mathrm{Ga}$ (e.g., ${ }^{68} \mathrm{Ga}$ DOTA analogs), regardless of their genetic background [5].

Advancing understanding of the genetic and metabolic drivers of PGL and their links to specific cell membrane characteristics of these tumors will aid in the selection of the most appropriate staging investigations and also provide the potential for identifying therapeutic targets. In particular, very high expression of somatostatin receptors will identify patients 
suitable for peptide receptor radionuclide therapy (PRRT), for example with ${ }^{177} \mathrm{Lu}$-DOTA-SSA, in the same way that ${ }^{123} \mathrm{I}$ MIBG has been used to identify expression of the norepinephrine transporter system and suitability for ${ }^{131}$ I-MIBG therapy. The high sensitivity of ${ }^{68} \mathrm{Ga}$-DOTA-SSA in the detection of these tumors as well as its potential "theranostic" application has led to rapid adoption of this technique, where available, for assessing PGL.

While availability of several radiopharmaceuticals offers novel diagnostic approaches for a medical team taking care of a PGL patient, it also presents several challenges. Although utilization of multiple radiotracers for localization, staging, and functional characterization of a PGL in a sequential pattern might improve the sensitivity and specificity of disease detection, this approach poses logistic issues for radiopharmaceutical production and supply, as well as having psychological and financial implications for the patient. Furthermore, despite using modern technology and devices that minimize radiation exposure, the potential radiation hazard of such a paradigm needs to be considered. However, such a paradigm has the potential to identify tumor heterogeneity that may have implications for clinical management.

An alternative approach to minimize radiation exposure could be to administer a "radiotracer cocktail" of different PET tracers with low individual activities to be captured later in one imaging session. Although conceptually attractive, the increase in background noise due to different radiotracers would likely reduce image quality and hence lesion detectability and would negate the possibility of determining which tracer was taken up by the tumor and therefore the potential for choice of radionuclide therapy agent if indicated. Further, the utilization of the radiotracer cocktail approach would negatively impact future studies aiming at establishing links between imaging phenotype, molecular genetic background, and clinical outcomes.

A potential solution to these challenges is to adopt a tailored approach using a diagnostic algorithm based on PGL location, biochemical phenotype, and any known genetic background, which is a strategy that has been recommended in recent guidelines [6,7]. Selection of the appropriate imaging pathway using such algorithms is, itself, somewhat challenging because it requires information that is not always readily available at the time of investigating a suspected PGL. Firstly, acquiring results of all the required tests may take weeks. Secondly, about $60-70 \%$ of PGL patients have no identifiable germline mutations and therefore, in these patients, imaging phenotype/genotype correlations are not yet fully established. A pragmatic approach would be to assess metastatic risk based on presence or absence of a positive family history and the size of the primary tumor and levels of methoxytyramine. Knowledge about biochemical phenotype is also crucial since PGLs with adrenergic phenotype are almost always located in the adrenal gland. Catecholamine profiles can usually be obtained within a week of initial work-up. In cases of an adrenergic phenotype, the size of any adrenal lesion on CT or MRI of the adrenal is the most important. If a tumor is more than $5-6 \mathrm{~cm}$, functional imaging should be added. For cases limited to the adrenal on anatomical imaging, ${ }^{123}$ I-MIBG scintigraphy would generally be the first nuclear imaging option as this is generally widely available and approved for this purpose. ${ }^{18} \mathrm{~F}$-FDOPA PET appears to be the most sensitive imaging tool for these tumors but is not routinely available at most imaging centers worldwide. The role of ${ }^{68} \mathrm{Ga}$-DOTA-SSA remains to be established in this setting but may replace MIBG as the test of first choice where available. For the noradrenergic biochemical phenotype the choice of imaging becomes more difficult. In this situation, a combination of anatomical or functional imaging is usually required. Based on genotype and anatomical staging, the following firstline strategies could be used based on recent research findings: known SDHB: ${ }^{68}$ Ga-DOTA-SSA PET/CT [8]; metastatic and sporadic PGL of unknown genotype: ${ }^{18} \mathrm{~F}$-FDOPA or ${ }^{68} \mathrm{Ga}$-DOTA-SSA PET/CT; head and neck PGL: ${ }^{68}$ Ga-DOTA-SSA PET/CT [9, 10]; sympathetic PGL: ${ }^{18}$ F-FDOPA PET/CT [11]. If the chosen imaging techniques is not well matched with the anatomical imaging, another radiopharmaceutical should be considered. Options include, particularly ${ }^{18} \mathrm{~F}-\mathrm{FDG} \mathrm{PET} / \mathrm{CT}$ for SDHx, sympathetic PGL or metastatic and sporadic PGL of unknown genotype, ${ }^{18} \mathrm{~F}$-FDOPA for head and neck PGL. The optimal sequencing of tracers in such a step-by-step approach remains to be determined by experienced multidisciplinary centers.

In conclusion, the ability to detect PGLs by molecular imaging goes hand in hand with its ability to characterize the molecular metabolomics and epigenetic features of these tumors [12]. The diversity of available radiopharmaceuticals is an advantage for the current management of patients, no matter which approach is used. It is expected that information provided by novel imaging studies, particularly the application of ${ }^{68} \mathrm{Ga}$-DOTA-SSA PET/CT to other groups of PHEO/PGL (e.g., sporadic metastatic and various hereditary ones), will offer promising new results that will provide a better understanding of the most efficient use of imaging to determine treatment options for this disease. The development of radiopharmaceuticals against new targets and the combination of PET and MRI (including spectroscopy) [13] will offer new options. Their utilization will, however, require a more expanded understanding of this disease at a molecular level that depends heavily on collaborative research programs between experienced centers that will provide a better understanding of the intimate interaction of imaging phenotype and genotype. 


\section{Compliance with ethical standards}

Conflicts of interest None.

\section{References}

1. Gross MD, Shapiro B, Thrall JH, Freitas JE, Beierwaltes WH. The scintigraphic imaging of endocrine organs. Endocr Rev 1984;5: 221-81. doi:10.1210/edrv-5-2-221.

2. Timmers HJ, Chen CC, Carrasquillo JA, Whatley M, Ling A, Eisenhofer G, et al. Staging and functional characterization of pheochromocytoma and paraganglioma by $18 \mathrm{~F}$-fluorodeoxyglucose (18F-FDG) positron emission tomography. J Natl Cancer Inst 2012;104:700-8. doi:10.1093/jnci/djs188.

3. Letouzé E, Martinelli C, Loriot C, Burnichon N, Abermil N, Ottolenghi $\mathrm{C}$, et al. SDH mutations establish a hypermethylator phenotype in paraganglioma. Cancer Cell 2013;23:739-52. doi: 10.1016/j.ccr.2013.04.018.

4. Lendvai N, Pawlosky R, Bullova P, Eisenhofer G, Patocs A, Veech $\mathrm{RL}$, et al. Succinate-to-fumarate ratio as a new metabolic marker to detect the presence of SDHB/D-related paraganglioma: initial experimental and ex vivo findings. Endocrinology 2014;155:27-32. doi:10.1210/en.2013-1549.

5. Taieb D, Kaliski A, Boedeker CC, Martucci V, Fojo T, Adler Jr JR, et al. Current approaches and recent developments in the management of head and neck paragangliomas. Endocr Rev 2014;35:795819. doi:10.1210/er.2014-1026.

6. Taieb D, Timmers HJ, Hindié E, Guillet BA, Neumann HP, Walz MK, et al. EANM 2012 guidelines for radionuclide imaging of phaeochromocytoma and paraganglioma. Eur J Nucl Med Mol Imaging 2012;39:1977-95. doi:10.1007/s00259-012-2215-8.

7. Lenders JW, Duh QY, Eisenhofer G, Gimenez-Roqueplo AP, Grebe SK, Murad MH, et al. Pheochromocytoma and paraganglioma: an endocrine society clinical practice guideline. J Clin Endocrinol Metab 2014;99:1915-42. doi:10.1210/jc.2014-1498.

8. Janssen I, Blanchet EM, Adams K, Chen CC, Millo CM, Herscovitch P, et al. Superiority of [68Ga]-DOTATATE PET/CT to other functional imaging modalities in the localization of SDHBassociated metastatic pheochromocytoma and paraganglioma. Clin Cancer Res 2015;21:3888-95. doi:10.1158/1078-0432.CCR-142751.

9. Kroiss A, Putzer D, Frech A, Decristoforo C, Uprimny C, Gasser $\mathrm{RW}$, et al. A retrospective comparison between (68)Ga-DOTATOC PET/CT and (18)F-DOPA PET/CT in patients with extraadrenal paraganglioma. Eur J Nucl Med Mol Imaging 2013;40: 1800-8. doi:10.1007/s00259-013-2548-y.

10. Janssen I, Chen CC, Taieb D, Patronas NJ, Millo CM, Adams KT, et al. 68Ga-DOTATATE PET/CT in the localization of head and neck paragangliomas compared to other functional imaging modalities and CT/MRI. J Nucl Med 2015.

11. Kroiss A, Shulkin BL, Uprimny C, Frech A, Gasser RW, Url C, et al. (68)Ga-DOTATOC PET/CT provides accurate tumour extent in patients with extraadrenal paraganglioma compared to (123)IMIBG SPECT/CT. Eur J Nucl Med Mol Imaging 2015;42:33-41. doi:10.1007/s00259-014-2892-6.

12. Hofman MS, Hicks RJ. Moving beyond "lumpology": PET/CT imaging of pheochromocytoma and paraganglioma. Clin Cancer Res 2015;21:3815-7. doi:10.1158/1078-0432.CCR-15-1073.

13. Varoquaux A, le Fur Y, Imperiale A, Reyre A, Montava M, Fakhry $\mathrm{N}$, et al. Magnetic resonance spectroscopy of paragangliomas: new insights into in vivo metabolomics. Endocr Relat Cancer 2015;22: M1-8. doi:10.1530/ERC-15-0246. 